\title{
Localized Delay Signals Detected by Synthetic Aperture Radar Interferometry and Their Simulation by WRF 4DVAR
}

\author{
Youhei Kinoshita $^{1, *}$ and Masato Furuya ${ }^{2}$ \\ ${ }^{1}$ Institute of Engineering Innovation, School of Engineering, The University of Tokyo, Tokyo, Japan \\ ${ }^{2}$ Department of Earth and Planetary Sciences, Hokkaido University, Sapporo, Japan
}

\begin{abstract}
Localized propagation delay signals associated with linealigned convective cells were detected by the Synthetic Aperture Radar Interferometry (InSAR) technique on 25 August 2010 in Niigata prefecture. The maximum amplitude of the signal reached up to $22.5 \mathrm{~cm}$, which was approximately equivalent to $29 \mathrm{~mm}$ anomaly in precipitable water vapor (PWV). The nationwide radar rainfall intensity captured the spatial distribution of hydrometeors on both land and sea, which was similar to that of the InSARderived water vapor field, suggesting that the convective cells were initiated on the Japan Sea to the west-southwest of the observation area. A numerical weather model (NWM) simulation with the grid spacing of $2.5 \mathrm{~km}$ reproduced line-aligned convective cells with $3 \mathrm{~cm}$ smaller maximum amplitude to that in InSAR. A NWM simulation that assimilates Global Navigation Satellite System (GNSS)-derived PWV data for four-dimensional variational assimilation enhanced the water vapor flux convergence at the surface, which improved the amplitude of the localized delay signals. The advantage of the unique water vapor observation by InSAR enabled us to assess the meso-gamma scale NWM reproducibility in terms of water vapor, which is one of the fundamental prognostic parameter for NWMs.
\end{abstract}

(Citation: Kinoshita, Y., and M. Furuya, 2017: Localized delay signals detected by synthetic aperture radar interferometry and their simulation by WRF 4DVAR. SOLA, 13, 79-84, doi: $10.2151 /$ sola.2017-015.)

\section{Introduction}

Water vapor is one of the most important parameter for precise short-range weather prediction but is also known to be difficult to observe due to its high variability in both space and time (Mateus et al. 2013). In the end of the twentieth century, the Global Navigation Satellite System (GNSS) has been demonstrated to be capable of estimating the precipitable water vapor (PWV) in the zenith direction (Bevis et al. 1992) with a precision comparable to that derived from radiosonde observations (Ohtani and Naito 2000). Although the recent development of the GNSS system contributed to improve the spatial resolution of the water vapor observation, the spatial resolution of the GNSS observation is a few tens of kilometers at best (Shoji et al. 2014), which is insufficient to resolve kilometer-scale phenomena such as individual convective cells. On the other hand, PWV mapping with $1 \mathrm{~km}$ resolution has been achieved by multi-spectrum infrared observations in the Moderate-Resolution Imaging Spectroradiometer and the Medium Resolution Imaging Spectrometer satellites ( $\mathrm{Li}$ et al. 2012). They provide high-resolution PWV maps in global with a recurrence period of 1-2 days and 3 days, but the observation is limited to the cloud-free condition. Detailed water vapor distributions under

Corresponding author: Youhei Kinoshita, Institute of Engineering Innovation, School of Engineering, The University of Tokyo, 2-11-16, Ya-yoi, Bunkyo-ku, Tokyo 113-8656, Japan. E-mail: ykinoshita@sogo.t.u-tokyo. ac.jp.

*Present affiliation:Research and Development Department, Remote Sensing Technology Center of Japan, 3-17-1, Tokyu REIT Toranomon Building, 3F, Minato-ku, Tokyo, Japan. E-mail: kinoshita_youhei@restec.or.jp. (C)2017, the Meteorological Society of Japan. extreme weather conditions such as a severe storm will often encounter the cloud-cover problems.

Interferometric Synthetic Aperture Radar (InSAR) is another microwave-based geodetic technique that can detect smallamplitude ground displacements with much higher spatial resolution than the GNSS system. InSAR image is called interferogram, and is derived by differencing the phase data in two SAR images acquired at different epochs. Initial interferogram consists of phase fringes due to the orbital separation, local topography, surface deformation, ionospheric and tropospheric propagation delay (Rosen et al. 2000). After the removal of the orbital contribution and the topographic effect, InSAR phase signals contain contributions due to ground deformation and atmospheric propagation effects including the ionospheric and tropospheric disturbance (e.g. Zebker et al. 1997). The tropospheric delay in InSAR is strongly related to the spatial heterogeneity of the integrated water vapor along radar line of sight. Therefore, in the absence of both of ground deformation and the ionospheric disturbance, InSAR can measure the spatial distribution of the integrated water vapor along the line-of-sight direction with the unprecedented spatial resolution (Hanssen et al. 1999). Kinoshita et al. (2013) detected the water vapor distribution during a heavy rain event in 2008 at middle Honshu, Japan, using emergency observation data of a Phased Array-type L-band Synthetic Aperture Radar (PALSAR), an L-band SAR sensor on board the Advanced Land Observing Satellite (ALOS). They performed numerical weather model (NWM) simulations to reproduce the localized water vapor signal detected by InSAR. In addition, Pichelli et al. (2015) performed a data assimilation experiment of the InSAR-derived PWV into the $1 \mathrm{~km}$ resolution mesoscale simulation at Rome (Central Italy), which showed statistically positive effects on the precipitation forecast.

The objectives of this study are first to demonstrate water vapor signals associated with convective cells by InSAR, and secondly to validate the impact of the GNSS data assimilation for improving the reproductivity of NWM simulations of the convective systems detected by InSAR in terms of the water vapor field.

\section{Data and model descriptions}

\subsection{InSAR data}

The SAR data used in this study were acquired by ALOS/ PALSAR launched by the Japan Aerospace Exploration Agency (JAXA) at 01:14 UTC on 25 August 2010 and at 01:14 UTC on 25 May 2010 from descending orbit (path 59, frame 2840). The perpendicular component of the baseline is $184.0 \mathrm{~m}$, which is short enough to keep interferometric coherence. The interferogram was generated with the GAMMA software (Werner et al. 2000). The orbital and topographic fringes were modeled using precise orbit data from JAXA and the $10 \mathrm{~m}$-mesh digital ellipsoidal height model generated by the GeoSpatial Information Authority of Japan, respectively. After subtracting orbital and topographic fringes from the initial interferogram, we used the minimum cost flow method (Constantini 1998) for phase unwrapping that could eliminate $2 \pi$ ambiguities in the phase image and quantify the displacements and propagation delays. The spatial resolution of the multi-looked InSAR image is approximately $40 \mathrm{~m}$. 


\subsection{Tropospheric delay}

Tropospheric delay observed by space geodetic systems such as InSAR and GNSS is caused by the refractivity that deviates from the value in vacuum due to the presence of air molecules. Atmospheric refractivity below the ionosphere can be represented as the following theoretically-derived equation (Thayer 1974)

$$
N=10^{-6}(n-1)=k_{1} \frac{P_{d}}{T}+k_{2} \frac{P_{v}}{T}+k_{3} \frac{P_{v}}{T^{2}},
$$

where $n$ represents refractive index, $T$ represents the absolute temperature in Kelvin $(\mathrm{K})$, and $P_{d}$ and $P_{v}$ represent dry air and water vapor partial pressure in Pascal $(\mathrm{Pa})$, respectively; $k_{1}, k_{2}$ and $k_{3}$ are experimentally-determined numerical constants of $k_{1}=$ $77.604(\mathrm{~K} / \mathrm{hPa}), k_{2}=64.79(\mathrm{~K} / \mathrm{hPa})$ and $k_{3}=3.776\left(\mathrm{~K}^{2} / \mathrm{hPa}\right)(\mathrm{Bevis}$ et al. 1992). The effects due to water droplet are not included in the above equation because they are minor contributor to the refractivity. In order to simulate the observed delay, the propagation delay $L_{e}$ from the satellite to the ground is derived by integrating the refractive index $n$ in equation (1) along the microwave path and can be expressed as,

$$
L_{e}=\int_{a t m}(n(\mathbf{r})-1) d s+\left(\int_{a t m} d s-\int_{v a c} d s\right)
$$

where atm indicates the Earth's atmosphere, $r$ indicates the coordinate of $n$, and $v a c$ indicates vacuum field. We use the NWM outputs in Eqs. (1) and (2). In Eq. (2), the first term in the right hand side represents the electromagnetic delay due to the change in the phase velocity and the second term represents the raybending effect (Hobiger et al. 2008). It is noted that the propagation delay effect derived from InSAR is the relative difference between the master and slave observations. Hence, water vapor signals should be included in both of the acquisition dates. In addition, the hydrostatic delay component expressed in the right first term in Eq. (1) can be negligible because the dry partial pressure $P_{d}$ is almost homogeneous within a few tens of kilometers. The effect of temperature is negligible not only because its spatial heterogeneity is small but also because the sensitivity of the delay amount to temperature is only $0.003 \mathrm{~cm}$ to $1 \mathrm{~K}$ difference. Therefore the InSAR phase delay primarily reflects the spatial difference of water vapor distributions between the two observation epochs.

\subsection{Numerical weather model}

We used the Weather Research and Forecasting (WRF) model version 3.4.1 (Skamarock et al. 2008) with Japan Meteorological Agency (JMA) Meso-Scale Model (MSM) objective analysis data and United States National Centers for Environmental Prediction high-resolution sea surface temperature data as the initial and boundary values. Initial and boundary conditions were derived from the MSM objective analysis data with the horizontal grid spacing of $5 \mathrm{~km}$ at the surface and $10 \mathrm{~km}$ above the surface 3 hourly from 18:00 UTC on 24 August to 03:00 UTC on 25 August. We performed the simulation with a single domain and we set the horizontal grid spacing of $2.5 \mathrm{~km}$ (Fig. 1a), which could resolve a whole picture of each convective cell dynamically. The horizontal grid numbers are 350 and 300 in north-south and east-west directions, respectively. The number of vertical layer was set to 50 , and the model top was $100 \mathrm{hPa}$. The 30 -arcsecond GTOPO30 model was used for the WRF topography. In WRF simulations, the WRF single-moment three-class microphysics scheme that includes the prognostic water substance variables of water vapor, cloud water/ ice, and rain/snow, the Dudhia shortwave radiation scheme and the rapid radiative transfer model long-wave radiation scheme were used. For the planetary boundary layer parametarization, the Mellor-Yamada-Janjic scheme was used. No cumulus parametarization was used. The simulation period was set from 18:00 UTC on 24 August 2010 to 03:00 UTC on 25 August 2010.

In addition, we performed the four-dimensional variational data assimilation (4DVAR) implemented in the WRF data assimilation (WRFDA, Huang et al. 2009) system with the aim of improving the reproductivity of numerical weather simulations. In this study we used the WRFDA version 3.4.1 and zenith total delay (ZTD) data derived from the Japanese dense GNSS network (GEONET) 3-hourly whose spatial density is approximately $25 \mathrm{~km}$ (Fig. 1b). ZTD data were converted to PWV using surface pressure and temperature data derived from surface meteorological observations. Hereafter, we will refer the WRF simulation without assimilation as the control run (CNTL) and with assimilation as 4DVAR. The grid spacings of the 4DVAR run were set to $10 \mathrm{~km}$ for the assimilation and $2.5 \mathrm{~km}$ for the convection-permitting simulation. The number of horizontal grids were set to $90 \times 80$ and $273 \times 245$ for coarser and finer domains, respectively. In the 4DVAR processing, we first initialized the MSM objective analysis data in the outer domain, and then conducted the assimilation process in this domain. The assimilation window was set from 18:00 UTC (simulation start time) on 24 August 2010 to 00:00 UTC on 25 August 2010. According to the fundamentals of data assimilation theory, this assimilation window of 6-hr at $10-\mathrm{km}$ grid spacing may not satisfy linearity. However, the 4DVAR outputs described later showed reasonable results, indicating the system worked appropriately. After the assimilation, we performed a 10-km WRF simulation and then downscaled the outputs to $2.5 \mathrm{~km}$ grid spacing to create initial and boundary conditions for the $2.5 \mathrm{~km}$ simulation. Finally, we performed the 2.5 $\mathrm{km}$ WRF simulation using downscaled initial and boundary fields from 18:00 UTC on 24 August. Other settings in 4DVAR were same as those in CNTL. Both CNTL and 4DVAR simulations were performed on 25 May 2010 to calculate propagation delay
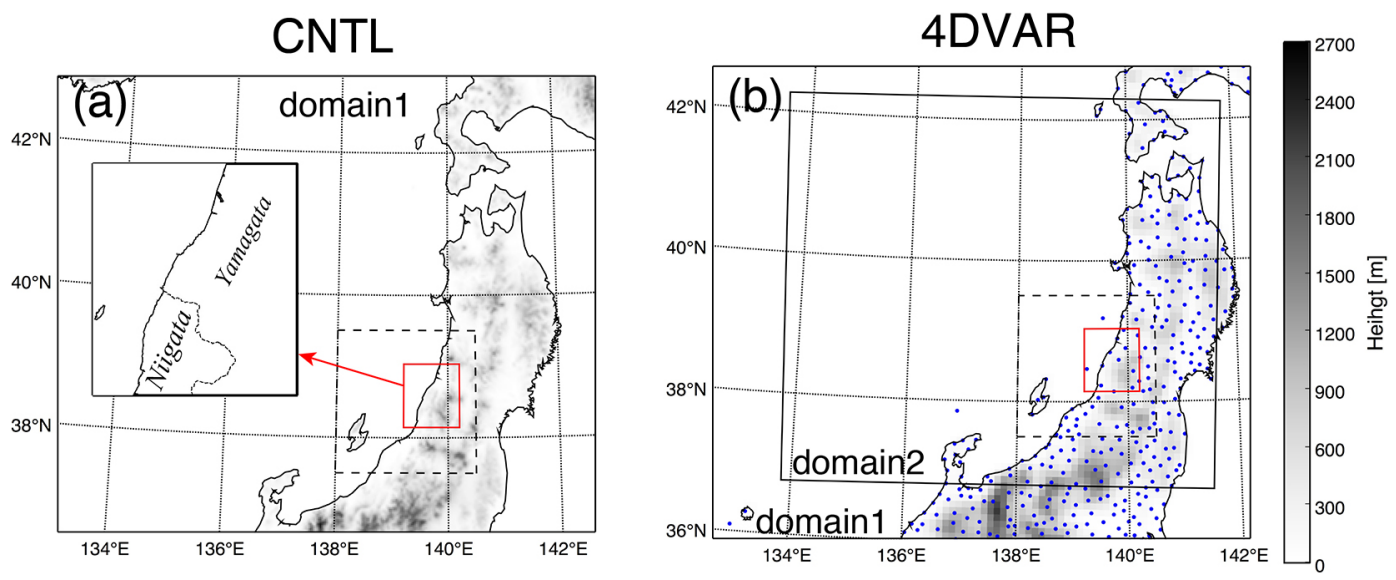

Fig. 1. (a) The WRF simulation domain in the CNTL simulation. The red rectangle indicates the area of Figs. 2 and 3 . The dashed black rectangle indicates the area of Fig. 5. (b) 4DVAR simulation domains. Blue dots indicate locations of GEONET stations used for the assimilation. 
differences for comparing with the observed InSAR image.

For assessing the reproductivity of CNTL and 4DVAR simulations, we calculated the propagation delay from WRF outputs based on Eqs. (1) and (2) with the ray-tracing method (Hobiger et al. 2008), which enables to directly compare model results with the derived interferogram. The modeled delay comparable with the InSAR image was derived by the difference of simulated delays on two epochs (in this case one is at 01:15 UTC on 25 August 2010 and the other is at 01:15 UTC on 25 May 2010).

\section{Results}

\subsection{Localized water vapor signals detected by InSAR}

The unwrapped interferogram captured clear phase changes that had the maximum amplitude of $22.5 \mathrm{~cm}$ in the line-of-sight direction and extended from WSW to ENE (Fig. 2 left). At first we validated that there were no surface deformation signal during the ALOS/PALSAR observation interval by comparing Fig. 2 with another InSAR image with the same spatial and temporal coverage and operational GNSS observation data, resulting in no significant surface deformation in the area (Supplements 1 and 2). In addition, we assume that the phase change in the interferogram is not due to the ionospheric effect but due to water vapor because the ionospheric effect in InSAR has spatially large wavelengths that are more than tens of kilometers (e.g. Gray et al. 2000). The detected water vapor signals included a few maxima with a horizontal scale of around $8 \mathrm{~km}$, whose characteristics resembles those detected by Kinoshita et al. (2013) in terms of the horizontal scale and amplitude, thus indicating the detected signals were indeed associated with convective cells. The corresponding zenith PWV can be approximately estimated by applying an equation $P W V_{s a}$ $=\Pi \cdot$ Delay $_{\text {sar }} \cdot \cos \theta$, where $\Pi$ indicates a conversion factor used for GNSS PWV estimation and $\theta$ indicates the incidence angle (Fornaro et al. 2015). Applying this equation, the observed InSAR image indicated the maximum anomaly of PWV up to $29 \mathrm{~mm}$ higher than the background PWV average around high PWV anomalies. The PWV field derived from the MSM objective analysis data and the GNSS observation at 00:00 UTC on 25 August 2010 did not show any localized increases corresponding to the localized PWV increase detected by InSAR (Supplement 3).

The rainfall intensity observed by the JMA operational radar network at 01:10 UTC on 25 August 2010 captured the convective systems with the rainfall intensity over $50 \mathrm{~mm} /$ hour, whose spatial distribution was similar to that detected by InSAR (Fig. 2 right).
It is noted that the detectable of weather radar is different from that of InSAR: InSAR detects the integrated tropospheric water vapor along the line-of-sight, whereas the weather radar detects the intensity of hydrometeors. Therefore the accordance between locations of the localized large-amplitude delay signal in InSAR and the high rainfall intensity of weather radar echo strongly suggests that a precipitation system with high rainfall intensity accompanies large amount of water vapor. On the other hand, there were no rainfall signals in the JMA radar rainfall intensity at 01:10 UTC on 25 May 2010 around the target area, thus suggesting that the InSAR-derived water vapor signals can be attributed to the high rainfall intensity on 25 August 2010.

\subsection{Numerical weather model simulations}

The CNTL simulation successfully reproduced convective cells, which initiated on the Japan Sea to the west of the study area, and then moved toward the ENE direction, and finally reached the coast (Fig. 3). The maximum PWV value of the convective cell reached up to $68.5 \mathrm{~mm}, 18.9 \mathrm{~mm}$ higher than the reference point $\mathrm{A}$. The CNTL simulation indicated that a number of convective cells were generated over the sea and passed through the coastline, and the lifetime of each convective cell was approximately an hour which is a typical lifetime of the single-cell (Szoke and Zipser 1986).

The 4DVAR simulation results are also shown in Fig. 3, which reproduced the convective systems as the CNTL simulation did. It is clear that the horizontal scale of each convective cell and the maximum PWV in the 4DVAR simulation were similar to those in the CNTL simulation. Clearly, in the 4DVAR simulation, there were a number of localized high PWV maxima that accompanied intense rainfall of over $30 \mathrm{~mm} / \mathrm{hr}$, indicating that these PWV maxima were associated with developed convective cells. On the other hand, in the CNTL simulation, many of PWV maxima indicated relatively weaker rainfall of $10 \mathrm{~mm} / \mathrm{hr}$ although a PVW maximum arrowed in Fig. 3 accompanied rainfall of over $30 \mathrm{~mm} / \mathrm{hr}$. Therefore, Fig. 3 suggests that the GNSS-PWV assimilation to the WRF simulation modified the PWV field in the initial condition and then increased the amount of water vapor in the convective cells.

Figure 4 shows the observed interferogram and estimated delay maps calculated from CNTL and 4DVAR outputs, respectively. These results clearly showed that the amplitude of reproduced convective cells improved in the assimilation experiment. These model simulations also indicated that localized delay signals were mainly caused by water vapor concentration, not by the
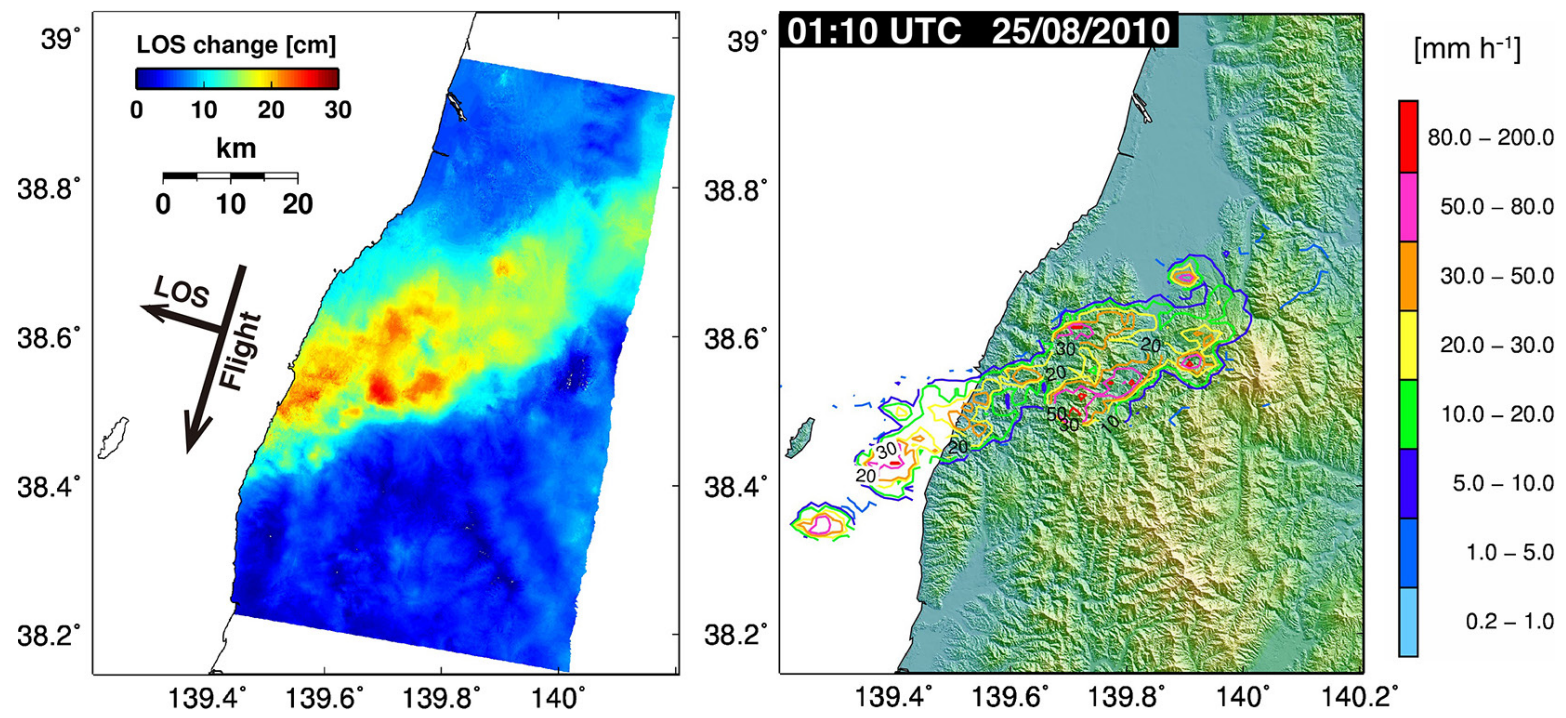

Fig. 2. (Left) The InSAR image derived from SAR data acquired at 01:14 UTC on 25 August 2010 (slave) and 25 May 2010 (master). The positive anomaly (red) indicates the existence of large amounts of water vapor at that point on the slave date. An average elevation angle within the image was $49.9^{\circ}$. (Right) Time series of the rainfall intensity distribution derived from the C-band weather radar around Japan. 
CNTL

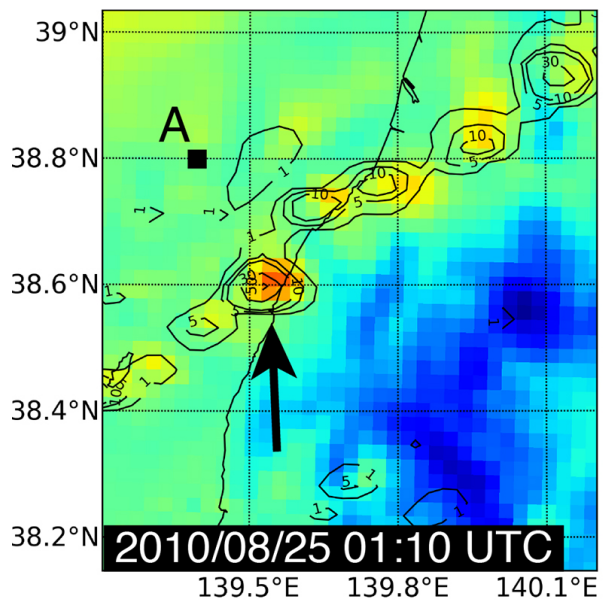

4DVAR
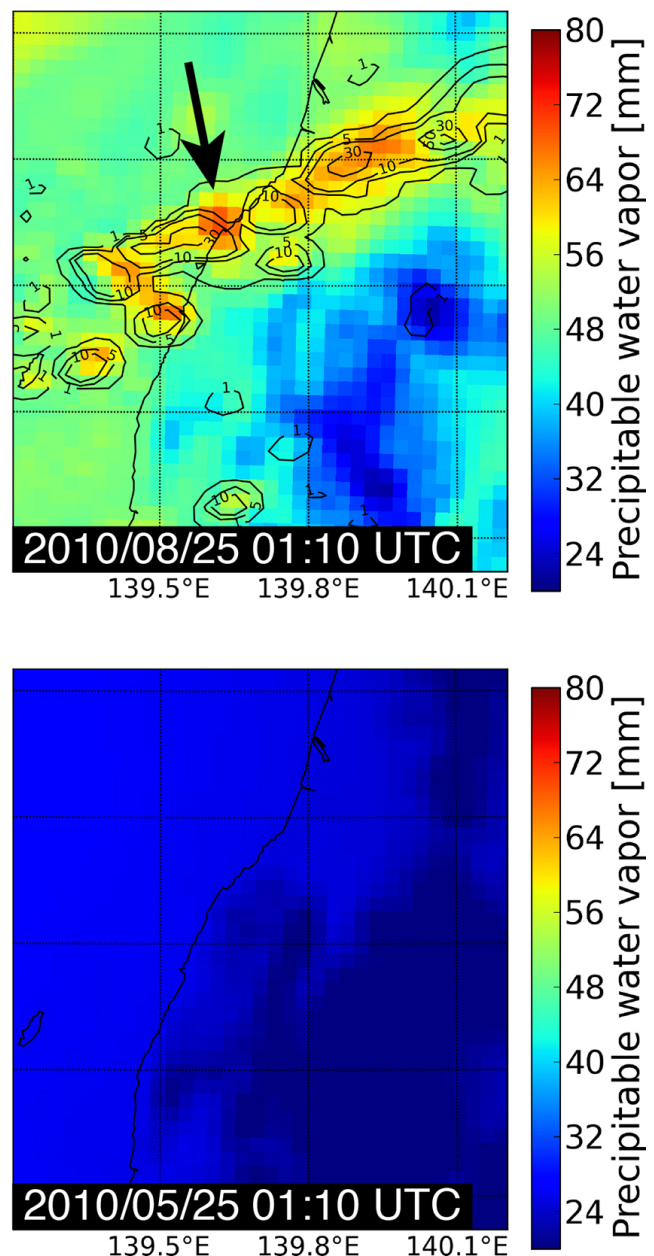

Fig. 3. Time series of the simulated PWV in the CNTL simulation (upper) and in the 4DVAR simulation (bottom), respectively. Contours indicate the simulated rainfall intensity with a unit of $\mathrm{mm} /$ hour. Arrows in upper images indicate PWV maxima. The symbol A (black square) indicates a reference location representing the background condition.

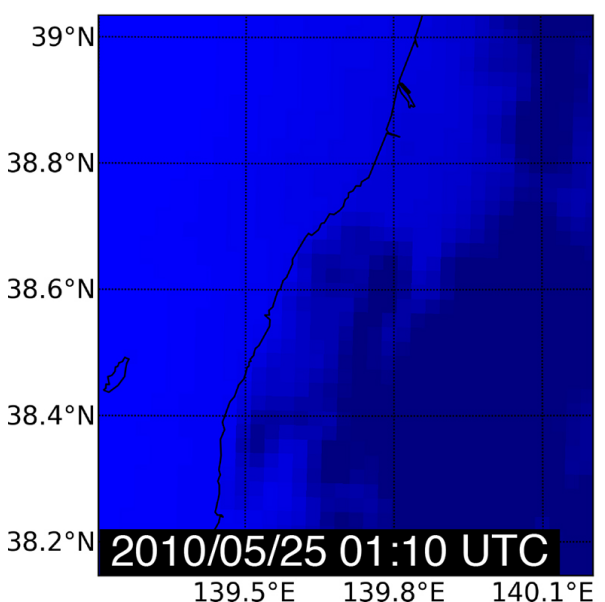

CNTL

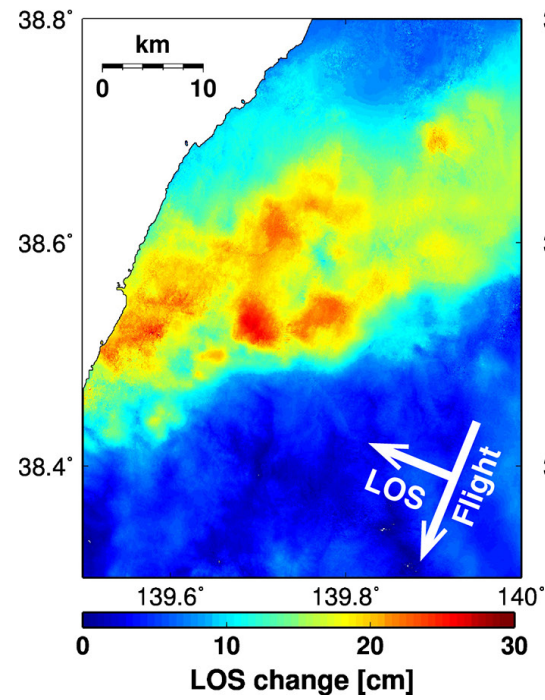

InSAR

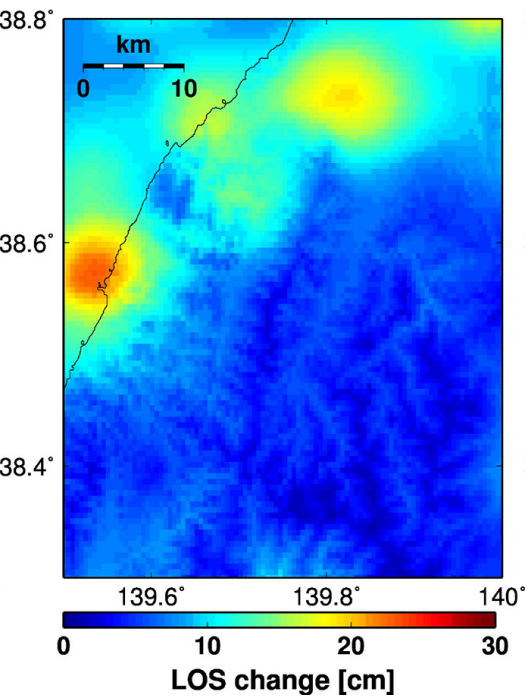

4DVAR

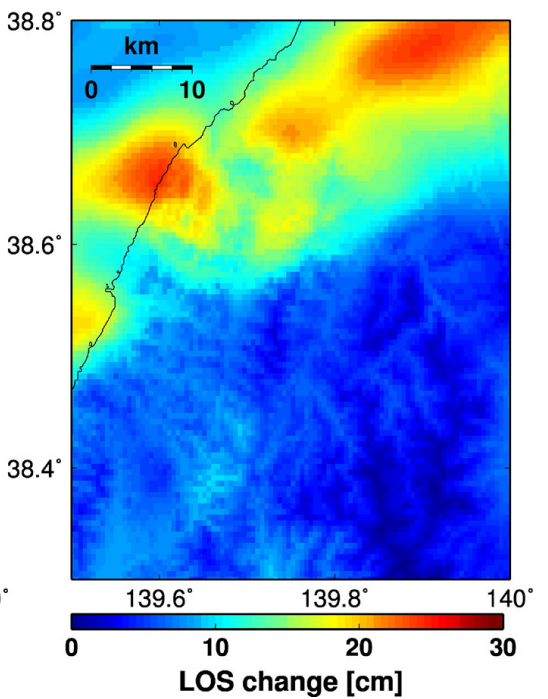

Fig. 4. (Left) The observed interferogram. (Center) The simulated propagation delay by the CNTL simulation at 01:15 UTC. (Right) The simulated propagation delay by the 4DVAR simulation at 01:15 UTC. 
dry air components represented in the first term in the right side of Eq. (1) (Supplement 4). The maximum amplitude in the CNTL simulation was $3 \mathrm{~cm}$ smaller than that in the observation and that in the 4DVAR simulation was $1 \mathrm{~cm}$ smaller than that in the observation. The phase noise of ALOS interferometry is approximately $3.3 \mathrm{~mm}$ in the line-of-sight direction (Sandwell et al. 2008). Therefore, these amplitude differences are significantly large and both CNTL and 4DVAR simulations underestimated propagation delay amounts relative to the observation. As is the case with the PWV distribution in Fig. 3, the CNTL simulation reproduced fewer localized large-amplitude signals than the observed interferogram. The 4DVAR simulation reproduced more localized largeamplitude signals than the CNTL simulation, getting more similar to the interferogram.

To investigate the assimilation impact on the improvement of the amplitude of reproduced convective cells, we calculated the divergence of the water vapor flux at the surface, which could contribute to the degree of the development of the convective cell. Figure 5 shows the 1 hour average divergence of the water vapor flux in CNTL and 4DVAR simulations from 00:10 UTC to 01:10 UTC, both of which indicate the presence of a strong convergence zone at the windward coast of the InSAR observation area. Model results indicated that most of the reproduced convective cells were initiated around the water vapor convergence zone. Therefore the strong water vapor convergence near the surface played an important role for the degree of the development of convective cells around the study area. With the use of the assimilation, water vapor convergence was enhanced. In addition, the convective available potential energy (CAPE) on the convergence zone $\left(139.5^{\circ} \mathrm{N}, 38.68^{\circ} \mathrm{E}\right)$ calculated from the 4DVAR output on $00: 10$ UTC was $1800 \mathrm{~J} / \mathrm{kg}$, indicating the unstable condition. Therefore the convergence enhancement of the water vapor flux in the 4DVAR simulation was one of the key factors for improving the model skill in this case, which was caused by the modified initial water vapor field through the four-dimensional data assimilation method with the GNSS PWV observation.

\section{Summary and discussions}

In this study we detected localized delay signals due to water vapor concentration on 25 August 2010 at Niigata prefecture by InSAR based on ALOS/PALSAR data. The InSAR image captured localized propagation delay signals associated with convective cells extending from WSW to ENE in a line shape. The maximum amplitude corresponded to $29 \mathrm{~mm}$ in PWV anomaly from the surrounding average. The rainfall intensity observed by the JMA operational radar network at 01:10 UTC on 25 August 2010 showed the spatial distribution of hydrometeors that was quite consistent with the InSAR-derived delay signals. Therefore the detected phase delay was very likely due to the water vapor associated with the rain band. The numerical weather simulation without the assimilation could reproduce the convective cells. The maximum propagation delay amount calculated from the CNTL run output with the ray-tracing method was $3 \mathrm{~cm}$ smaller than the detected delay signal in the InSAR image and the number of localized delay maxima were fewer than the observed interferogram. The 4DVAR run with GNSS PWV data assimilation was conducted with the same grid spacing, demonstrating the improvement in the delay amplitude of the reproduced convective cells that were $2 \mathrm{~cm}$ closer to the observed one than that in the CNTL run. The 4DVAR simulation reproduced localized signals with large amplitude than the CNTL simulation. In addition, the number of localized delay signals with large amplitude increased in the 4DVAR simulation. The improvement of the WRF reproducibility by data assimilation was due to the enhancement of the surface water vapor convergence in the Japan sea west of the study area.

The precise modeling of the water vapor propagation delay is one of the important challenge in InSAR toward the higher detection accuracy of surface deformation. Our result showed not only the effectiveness of the 4DVAR assimilation for the numerical weather simulation, but also highlighted the possibility to correct the turbulent component of the InSAR tropospheric propagation delay by the numerical weather model for surface deformation studies. To investigate the effectiveness of the numerical weather model with the data assimilation, further studies that, for example, assimilate other meteorological variables like wind and temperature to reproduce InSAR-derived meteorological phenomena should be conducted.

Our study showed the unique ability of InSAR to observe water vapor distributions with an unprecedented spatial resolution. The advantage of InSAR can be applied not only to elucidating a mechanism of severe storm events but also to investigating the thermodynamic characteristics of meso-scale phenomena like mountain waves and convective rolls in the boundary layer.

The poor temporal resolution is one of the biggest limitations in the SAR system for the meteorological application. However, Mateus et al. (2013) mentioned that the merging of water vapor maps obtained by combination of different spaceborne SAR mis-
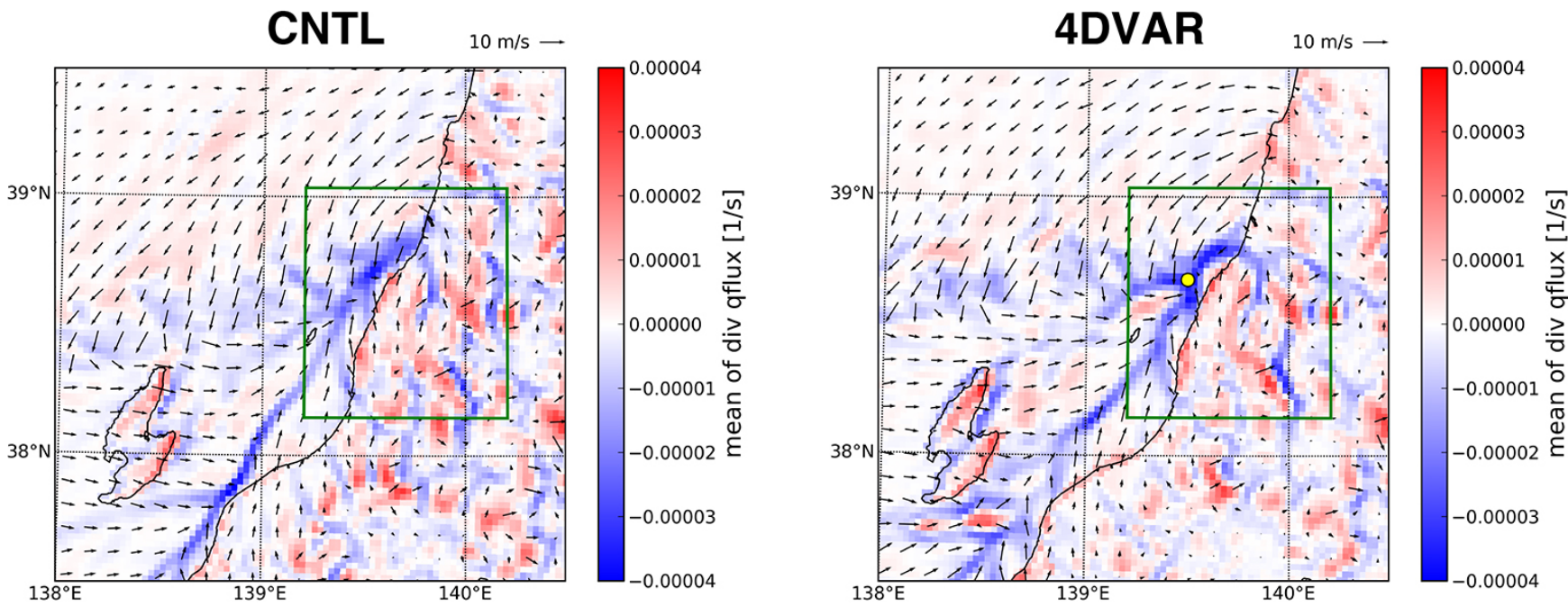

Fig. 5. The horizontal wind distribution at 01:10 UTC on 25 August 2010 and the 1 hour average of the water vapor flux divergence at the surface derived from the CNTL simulation (left) and the 4DVAR simulation (right) from 00:10 UTC to 01:10 UTC. The blue color indicates the water vapor flux convergence which could contribute to the development of the convection. Green rectangles indicate the area of Figs. 2 and 3 . The yellow circle in the right figure indicates the CAPE calculation point. 
sions such as Sentinel-1 and ALOS-2 missions has the possibility for improving the temporal resolution with a sampling period of a few days or better.

\section{Acknowledgments}

The PALSAR level 1.0 data in this study were provided by the PALSAR Interferometry Consortium to Study our Evolving Land surface (PIXEL) under cooperative research contracts between the Earthquake Research Institute at the University of Tokyo and JAXA, and by JAXA through the arrangement of ALOS-2 Fourth Research Announcement (PI:1286). PALSAR data belong to JAXA and the Ministry of Economy, Trade and Industry. The weather radar data and MSM data were acquired from the grid point value archive site (http://database.rish.kyoto-u.ac.jp/arch/ jmadata/) managed by the Research Institute for Sustainable Humanosphere, Kyoto University. We acknowledge two anonymous reviewers for their helpful comments.

Edited by: S. Nishizawa

\section{Supplements}

Supplement 1: An InSAR image derived from SAR data acquired at 13:07 UTC on 2 May 2010 and 17 September 2010. There are no significant displacement signals in this image. This interval covers the whole time interval of the InSAR observation in Fig. 2.

Supplement 2: Surface displacement in the SAR line-of-sight direction derived from GEONET F3 daily coordinate data between 2 May 2010 and 17 September 2010. A green box indicates the area of Figs. 2 and 3.

Supplement 3: PVW distributions derived from MSM data and GEONET zenith tropospheric delay data (colored circles) at 18:00 UTC on 25 August 2010.

Supplement 4: Simulated propagation delays of total amount (left), delays caused by water vapor (center) and delays caused by dry air (right). Upper images are derived from the CNTL simulation and lower images from the 4DVAR simulation.

\section{References}

Bevis, M., S. Businger, T. A. Herring, C. Rocken, R. A. Anthes, and R. H. Ware, 1992: GPS meteorology: Remote sensing of atmospheric water vapor using the Global Positioning System. J. Geophys. Res., 97, 787-801.

Costantini, M., 1998: A novel phase unwrapping method based on network programming. IEEE TGRS, 36, 813-821.

Fornaro, G., N. D’Agostino, R. Giuliani, C. Noviello, D. Reale, and S. Verde, 2015: Assimilation of GPS-derived atmospheric propagation delay in DInSAR data processing. IEEE JSTARS, 8, 784-799.

Gray, A. L., K. E. Mattar, and G. Sofko, 2000: Influence of ionospheric electron density fluctuations on satellite radar interferometry. Geophys. Res. Lett., 27, 1451-1454.

Hanssen, R. F., T. M. Weckwerth, H. A. Zebker, and R. Klees, 1999: High-resolution water vapor mapping from interferometric radar measurements. Science, 283, 1297-1299.
Hobiger, T., R. Ichikawa, Y. Koyama, and T. Kondo, 2008: Fast and accurate ray-tracing algorithms for real-time space geodetic applications using numerical weather models. $J$. Geophys. Res., 113, D20302.

Huang, X.-Y., and co-authors, 2009: Four-dimensional variational data assimilation for WRF: Formulation and preliminary results. Mon. Wea. Rev., 137, 299-314.

Kinoshita, Y., M. Shimada, and M. Furuya, 2013: InSAR observation and numerical modeling of the water vapor signal during a heavy rain: A case study of the 2008 Seino event, central Japan. Geophys. Res. Lett., 40, 4740-4744.

Li, Z. W., W. B. Xu, G. C. Feng, J. Hu, C. C. Wang, X. L. Ding, and J. J. Zhu, 2012: Correcting atmospheric effects on InSAR with MERIS water vapour data and elevationdependent interpolationmodel. Int. J. Geophys., 189, 898910.

Mateus, P., G. Nico, and J. Catalão, 2013: Can spaceborne SAR interferometry be used to study the temporal evolution of PWV?. Atmos. Res., 119, 70-80.

Ohtani, R., and I. Naito, 2000: Constructing accurate maps of atmospheric water vapor by combining interferometric synthetic aperture radar and GNSS observations. J. Geophys. Res., 105, 917-929.

Pichelli, E., R. Ferretti, D. Cimini, G. Panegrossi, D. Perissin, N. Pierdicca, F. Rocca, and B. Rommen, 2015: InSAR water vapor data assimilation into mesoscale model MM5: Technique and pilot study. IEEE JSTARS, 8, 3859-3875.

Rosen, P. A., S. Hensley, I. R. Joughin, F. K. Li, S. N. Madsen, E. Rodrïguez, and R. M. Goldstein, 2000: Synthetic aperture radar interferometry. IEEE, 88, 333-382.

Sandwell, D. T., D. Myer, R. Mellors, M. Shimada, B. Brooks, and J. Foster, 2008: Accuracy and resolution of ALOS interferometry: Vector deformation maps of the father's day intrusion at Kilauea. IEEE TGRS, 46, 3524-3534.

Shoji, Y., H. Yamauchi, W. Mashiko, and E. Sato, 2014: Estimation of local-scale precipitable water vapor distribution around each GNSS station using slant path delay. SOLA, 10, 29-33.

Skamaroch, W. C., J. B. Klemp, J. Dudhia, D. O. Gill, D. M. Barker, M. G. Duda, X. Y. Huang, W. Wang, and J. G. Powers, 2008: A description of the Advanced Research WRF version 3. NCAR Tech. Note, NCAR/TN-475+STR, 113 pp. (Available online at http://www.mmm.ucar.edu/wrf/ users/docs/arw_v3.pdf, accessed on 24 March 2017).

Szoke, E. J., and E. J. Zipser, 1986: A radar study of convective cells in mesoscale systems in GATE. Part II: Life cycles of convective cells. J. Atmos. Sci., 43, 199-218.

Thayer, G. D., 1974: An improved equation for the radio refractive index of air. Radio Sci., 9, 803-807.

Werner, C., U. Wegmüller, T. Strozzi, and A. Wiesmann, 2000: SAR and interferometric processing software. Proc. ERSENVISAT Symp.

Zebker, H. A., P. Rosen, and S. Hensley, 1997: Atmospheric effects in interferometric synthetic aperture radar surface deformation and topographic maps. J. Geophys. Res., 102, 7547-7563.

Manuscript received 1 December 2016, accepted 24 March 2017 SOLA: https://www.jstage.jst.go.jp/browse/solal 